\title{
New Hybrid Organic-Inorganic Thin Films by Molecular Layer Deposition for Rechargeable Batteries
}

\author{
Jian Liu $^{1 *}$ and Jiajun Wang ${ }^{2 *}$ \\ ${ }^{1}$ School of Engineering, Faculty of Applied Science, the University of British Columbia, Kelowna, BC, Canada, ${ }^{2}$ MIIT Key \\ Laboratory of Critical Materials Technology for New Energy Conversion and Storage, School of Chemistry and Chemical \\ Engineering, Harbin Institute of Technology, Harbin, China
}

The design of multifunctional thin films holds the key to manipulate the surface and interface structure of the electrode and electrolyte in rechargeable batteries and achieve desirable performance for various applications. Molecular layer deposition (MLD) is an emerging thin-film technique with exclusive advantages of depositing hybrid organicinorganic materials at a nanoscale level and with well tunable and unique properties that conventional thin films might not have. Herein, we provide a timely mini-review on the most

OPEN ACCESS

Edited by:

Sheng S. Zhang,

United States Army Research Laboratory, United States

Reviewed by: Xiulin Fan,

Zhejiang University, China Wen Yang,

Beijing Institute of Technology, China

*Correspondence: Jian Liu

Jian.liu@ubc.ca

Jiajun Wang

Jiajunhit@hit.edu.cn

Specialty section:

This article was submitted to Electrochemical Energy Conversion and Storage,

a section of the journal Frontiers in Energy Research

Received: 09 February 2021 Accepted: 06 May 2021

Published: 21 May 2021

Citation:

Liu J and Wang J (2021) New Hybrid Organic-Inorganic Thin Films by Molecular Layer Deposition for

Rechargeable Batteries.

Front. Energy Res. 9:665884. doi: 10.3389/fenrg.2021.665884 recent progress in the surface chemistry and MLD process of novel hybrid organicinorganic thin films and their applications as the anode, cathode, and solid electrolytes in lithium-ion batteries. Perspectives for future research in designing new MLD process and precursors, enriching MLD material library, and expanding their potential applications in other energy storage systems, are discussed at the end.

Keywords: molecular layer deposition, hybrid thin film, surface coating, lithium batteries, self-limiting growth

\section{INTRODUCTION}

Rechargeable batteries play an essential role in many applications nowadays, from portable devices and electric vehicles to miniaturized and intelligent systems, such as wearable devices, the Internet of things, micro-electromechanical system. The ever-increasing demand for high-performance batteries requires continuous materials innovation to address the limitation in current rechargeable batteries. In particular, many pressing issues in batteries, such as degradation, safety, gas evolution, etc., are closely associated with the structure and properties of the electrode and electrolyte interface (Xu et al., 2018; Banerjee et al., 2020; Rowden and GarciaAraez, 2020). It is well recognized that being able to manipulate the interface, often at the nanoscale level, is a key to alleviate unwanted side reactions and improve the overall performance of batteries, particularly at extreme conditions, such as high temperatures, high voltages, fast charging, etc. (Chen et al., 2010; Zhao et al., 2021).

Over the past decades, atomic layer deposition (ALD) has been demonstrated as a promising thin film deposition technique to tailor the electrode-electrolyte interface in liquid and all-solid-state lithium-ion batteries (LIBs) (Knoops et al., 2012; Zhao et al., 2018b; Zhao et al., 2021). Distinguished from other techniques, ALD allows uniform and nanoscale coating of functional inorganic thin films (metal oxides, phosphates, fluorides, and nitrides) at the electrode-electrolyte interface in LIBs to suppress undesirable phenomena, such as detrimental surface and near-surface phase transition (Yan et al., 2018), electrode volume change (Xiao et al., 2011; Lotfabad et al., 2014), transitional metal dissolution (Scott et al., 2011), etc. Such success has stimulated the industrial pilot study of using a high-throughput continuous particle ALD process to achieve surface modification on large-scale 


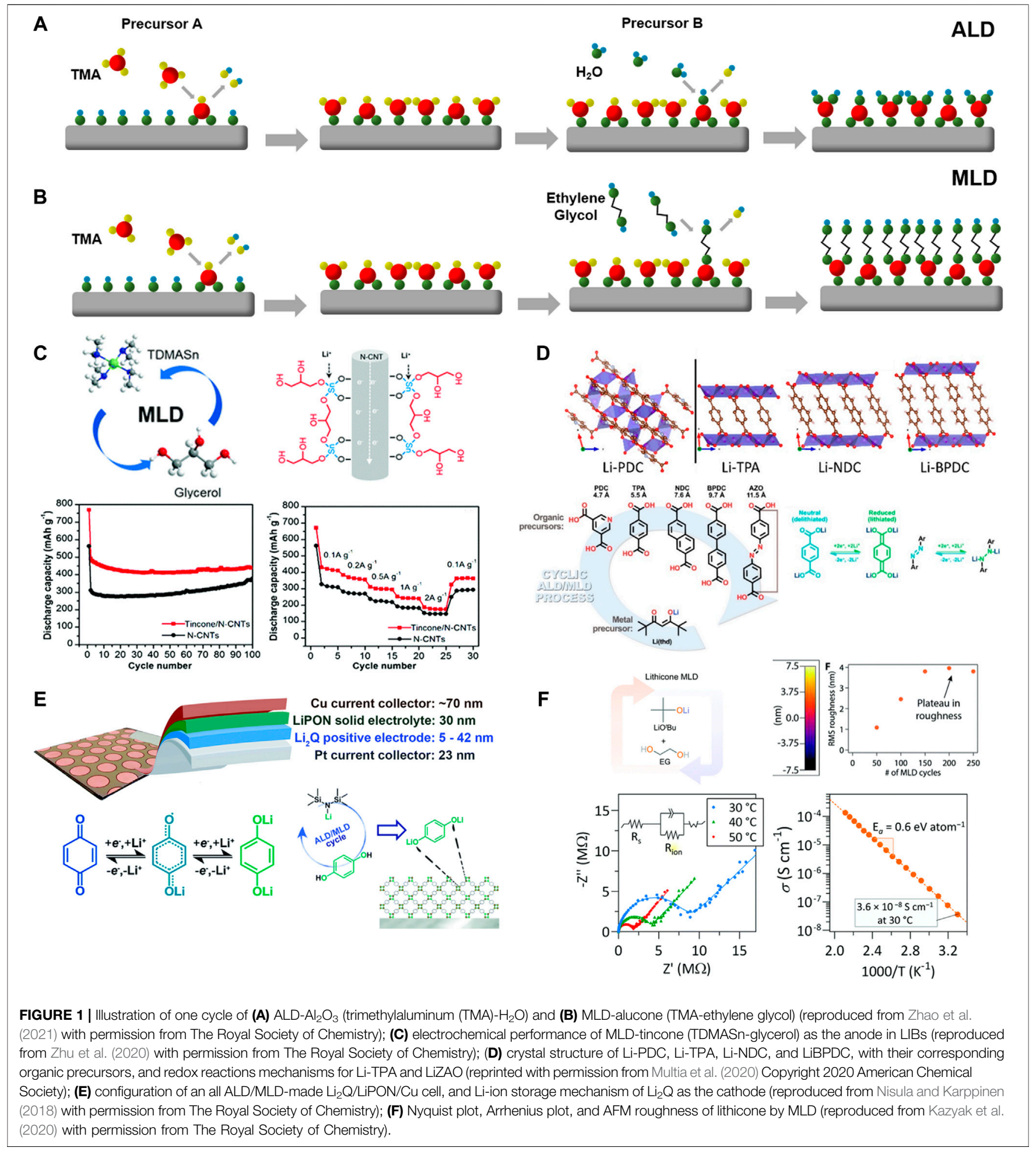

battery materials (Weimer, 2019). Besides application in transitional LIBs, ALD has been recently demonstrated for fabricating three-dimensional (3D) microbatteries (Pearse et al., 2017; Liu et al., 2018; Pearse et al., 2018). 3D microbatteries allow significantly increased surface area of active materials in the limited footprint as planar thin-film batteries and thus provide high energy and power densities (Figure 1A). However, manufacturing 3D microbatteries has been challenging due to the strict requirements on the uniformity and pinhole-free deposition of the anode, solid electrolyte, and cathode layers on high-surface-area substrates (Oudenhoven et al., 2011; Roberts et al., 2011; Liu et al., 2020), 
and only become possible with the advances in new ALD process development for these electrode and electrolyte materials. Therefore, it is vital to design and develop novel functional thin films with unique properties because it will provide numerous opportunities for enabling better battery technologies.

While ALD can only deposit inorganic materials, molecular layer deposition (MLD) has emerged as a thin film technique that resembles the benefits of ALD in controlling film thickness, uniformity, conformity, and crystallinity, but is able to deposit hybrid organic-inorganic materials, namely "metalcone" (George et al., 2009; Lee et al., 2013). This is achieved by incorporating an organic fragment in the thin films by pairing metal precursors with organic reactants (Figure 1B), instead of water as in ALD (Figure 1A), during the sequential and self-limiting deposition process. One MLD cycle consists of four steps. During the first step of the MLD process, metalorganic precursor (such as trimethylaluminum-TMA) is introduced into the chamber and react with all surface functional groups (e.g., hydroxyl group) through ligand exchange. In the second step, the excessive precursor and any byproducts are entirely removed by inert gas purging. In the third step, an organic precursor (e.g., ethylene glycol-EG) is pulsed into the chamber to react with metalorganic ligands to convert the surface back to hydroxyl groups. Once the reaction is complete, the residual organic precursor and byproducts are pumped out using gas purging.

The incorporation of organic fragments technically opens unlimited possibilities for engineering the structure of metalcone at the molecular level and tailoring their mechanical, electrical, optical, and electrochemical properties for energy storage and conversion systems (Sundberg and Karppinen, 2014; Meng, 2017). For example, MLD alucone has been applied as a flexible surface coating layer on the various anode $\left(\mathrm{Si}, \mathrm{SnO}_{2}, \mathrm{Li} / \mathrm{Na} / \mathrm{Zn}\right.$ metals $)$ and cathode in rechargeable $\mathrm{Li}, \mathrm{Na}$, and $\mathrm{Zn}$ batteries (Piper et al., 2014; Zhao et al., 2018a; He and Liu, 2020), and been found to outperform its ALD $\mathrm{Al}_{2} \mathrm{O}_{3}$ counterpart in stabilizing the electrode-electrolyte interfaces owing to the added benefits from the organic fragment. For example, alucone coating, deposited by using trimethylaluminum (TMA) and glycerol (GL) at $140^{\circ} \mathrm{C}$, was found to improve the cycling stability, rate, and Coulombic efficiency of nano-Si composite electrodes (Piper et al., 2014). Alucone was proven to be robust and resilient to accommodate the large volume change of $\mathrm{Si}$ and maintain an intimate network for fast ionic and electron conduction (Ma et al., 2015; Son et al., 2017), while conventional metal oxides are not suitable for largevolume-change materials due to mechanical failure at high stresses. Therefore, as a surface coating on the anode, the MLD films should have good uniformity and coverage on the electrode, good Li-ion conductivity upon lithiation, and excellent toughness and flexibility to tolerate repeated stress. The properties of MLD alucone might be tuned by precisely adjusting the organic reactants during the deposition process (Lee et al., 2013). For Li metal anode, alucone coating (10-25 cycles) showed improved stripping and plating performance, stabilized polarization curves, lowered internal resistance, and prolonged lifetime, compared with $\mathrm{Al}_{2} \mathrm{O}_{3}$-protected or bare $\mathrm{Li}$ metal (Zhao et al., 2018a; Chen et al., 2018). The highly cross- linked structure in alucone coating with good mechanical properties and flexibility could effectively suppress dendrite formation, maintain continuous solid electrolyte interphase (SEI), and reduce the reactions and penetration of electrolyte with $\mathrm{Li}$ metal. MLD-alucone has also been applied as surface coating on P2-type $\mathrm{Na}_{0.66} \mathrm{Mn}_{0.9} \mathrm{Mg}_{0.1} \mathrm{O}_{2}$ (NMM) cathode in $\mathrm{Na}$ ion batteries, in comparison with $\mathrm{Al}_{2} \mathrm{O}_{3}$ coating and no coating. The alucone coated NMM cathode exhibited a 96\% capacity retention, compared to pristine (75\%) and $\mathrm{Al}_{2} \mathrm{O}_{3}$-coated $(71 \%)$ $\mathrm{NMM}$, after 100 cycles between 2 and $4.5 \mathrm{~V}$ at $1^{\circ} \mathrm{C}$ (Kaliyappan et al., 2020). Besides the mechanical robustness, the more electronically conductive nature of the alucone thin film than alumina was essential to improved rate capability and benefited from the carbon linkers in the alucone film. Therefore, different from the surface coating on the anode, MLD film coating on the cathode should primarily consider electronic conductivity and uniformity while emphasizing mechanical properties. Consequently, we believe that MLD provides a new opportunity to innovate novel thin-film materials to catalyze energy storage and conversion research.

Despite the great promise, hybrid organic-inorganic materials and their MLD surface chemistry and process are minimal, unlike the rich ALD material library. Many possible MLD materials remain unexplored, probably due to challenges associated with the selection of MLD precursors and the development of the MLD process. Excitedly, several proofof-concept MLD materials as the battery components, i.e., anode, cathode, and solid electrolytes, have been reported and demonstrated recently. As such, this mini-review is intended to provide a timely summary of the recent progress in MLD surface chemistry and process development for hybrid organic-inorganic materials, their applications and potentials in rechargeable batteries, and the intuitions learned so far to guide future multifunctional thin film design by MLD.

\section{NEW MLD THIN FILMS DEMONSTRATED AS ACTIVE BATTERY MATERIALS}

Early metalcone materials, i.e., titanicone (Van De Kerckhove et al., 2016), vanadicone (Van De Kerckhove et al., 2017), developed by MLD were electrochemically inactive toward Liion storage as the deposited state and required post-annealing to convert the metalcone into metal oxide-based materials to present Li-ion storage performance. However, the harish post-annealing process caused the damage of the thin film uniformity and usually led to the formation of island structures (Kazyak et al., 2017), making them unsuitable for fabricating $3 \mathrm{D}$ microbatteries. As shown in previous ALD works (Pearse et al., 2018), the thin films need to be electrochemically functional so that they can be directly applied to fabricate $3 \mathrm{D}$ microbatteries. To satisfy $3 \mathrm{D}$ microbattery requirements, the MLD thin films should process high electrochemical activity toward Li-ion storage or transport, have high uniformity and conformity on high-aspect-ratio substrates, and relatively straightforward deposition process and receipts. 
TABLE 1 | Summary of MLD receipts, growth behaviors, and applications of metalcone films.

\begin{tabular}{|c|c|c|c|c|c|c|c|c|}
\hline $\begin{array}{l}\text { Battery } \\
\text { component }\end{array}$ & MLD film & $\begin{array}{c}\text { Metal } \\
\text { precursor }\end{array}$ & $\begin{array}{l}\text { Organic } \\
\text { reactant }\end{array}$ & $\begin{array}{l}\text { Deposition } \\
\qquad \mathrm{T}\left({ }^{\circ} \mathrm{C}\right)\end{array}$ & GPC (Å) & $\begin{array}{c}\text { Post } \\
\text { annealing }\end{array}$ & Application & References \\
\hline \multirow[t]{10}{*}{ Anode } & Tincone & TDMASn & $\mathrm{GL}$ & 100 & 2.5 & No & LIBs & Zhu et al. (2020) \\
\hline & $\mathrm{Li}_{2} \mathrm{TP}$ (or Li-TPA) & Li(thd) & TPA & $200-240$ & 3.0 & No & LIBs & Nisula and Karppinen. (2016) \\
\hline & $\mathrm{Li}_{2} \mathrm{TP}-\mathrm{NH}_{2}$ & Li(thd) & TPA-NH ${ }_{2}$ & 200 & 3.6 & No & LIBs & Heiska et al. (2020) \\
\hline & Li-NDC & Li(thd) & NDC & 220 & 2.3 & No & LIBs & Multia et al. (2020) \\
\hline & Li-BPDC & Li(thd) & BPDC & 240 & 7.0 & No & LIBs & Multia et al. (2020) \\
\hline & Li-PDC & Li(thd) & PDC & 220 & 2.5 & No & LIBs & Multia et al. (2020) \\
\hline & $\mathrm{Li}-\mathrm{AZO}$ & Li(thd) & ZAO & 270 & 7.0 & No & LIBs & Multia et al. (2020) \\
\hline & Titanicone & TDMAT & $\mathrm{GL}$ & $80-160$ & $0.9-0.2$ & Yes & LIBs & Van De Kerckhove et al. (2016) \\
\hline & Magnesicone & $\mathrm{Mg}(\mathrm{MeCp})_{2}$ & EG or $\mathrm{GL}$ & $100-250$ & $2-3$ & Yes & N/A & Kint et al. (2020) \\
\hline & Ti-based maleic acid & $\mathrm{TiCl}_{4}$ & MA & $140-280$ & $1.42-0.16$ & No & N/A & Cao et al. (2019) \\
\hline \multirow[t]{5}{*}{ Cathode } & Vanadicone & TEMAV & $\mathrm{GL}$ & $80-180$ & $1.2-1.5$ & Yes & LIBs & Van De Kerckhove et al. (2017) \\
\hline & $\mathrm{Li}_{2} \mathrm{Q}$ & LiHMDS & $\mathrm{HQ}$ & 160 & - & No & LIBs & Nisula and Karppinen. (2018) \\
\hline & Manganicone & $\mathrm{Mn}(\mathrm{EtCp})_{2}$ & EG & 160 & 1 & Yes & N/A & Bergsman et al. (2019) \\
\hline & $\begin{array}{l}\text { Mn-based } \\
\text { hybrid film }\end{array}$ & $\mathrm{Mn}(\mathrm{thd})$ & TPA & 200 & $1-2$ & Yes & N/A & $\begin{array}{l}\text { Ahvenniemi and Karppinen. } \\
\text { (2016) }\end{array}$ \\
\hline & Co-based hybrid film & $\begin{array}{l}\text { Co. }(\text { acac })_{3} \text { or } \\
\text { Co.(thd })_{2}\end{array}$ & TPA & 200 & $1-2$ & Yes & N/A & $\begin{array}{l}\text { Ahvenniemi and Karppinen. } \\
\text { (2016) }\end{array}$ \\
\hline Solid & Lithicone & $\mathrm{LiO}^{t} \mathrm{Bu}$ & EG & 135 & 2.6 & Yes & LIBs & Kazyak et al. (2020) \\
\hline electrolyte & LPDO & $\mathrm{LiO}^{t} \mathrm{Bu}$ & PD & $150-200$ & $0.15-0.23$ & No & N/A & Wang et al. (2020) \\
\hline
\end{tabular}

TDMASn, tetrakis (dimethylamino) tin (IV); GL, glycerol; Li 2 TP, lithium terephthalate; thd, 2,2,6,6-tetramethyl-3,5-heptanedionate; TPA, terephthalic acid; TPA-NH 2 -2, aminoterephthalic acid; PDC, pyridinedicarboxylic acid; NDC-2,6-naphthalenedicarboxylic acid; BPDC, 4,4'-biphenydicarboxylic acid; ZAO, 4,4'-azobenzenedicarboxylic acid; LiO'Bu, Lithium tertbutoxide; EG, ethylene glycol; TDMAT, tetrakisdimethylaminotitanium; Mg(MeCp); $;$ TEMAV, tetrakisethylmethylaminovanadium; LiHMDS, lithium bis(trimethylsilyl)amide; HQ, hydroquinone; LPDO, lithium propane dioxide; PD, propanediol; Mn(EtCp)2, bis(ethylcyclopentadienyl)manganese; maleic acid, MA. N/A, This new MLD material is presumably for LIB applications but not demonstrated yet.

Alloying-type anode materials (e.g., Sn, Si) are known for their high specific capacities and their obvious drawback of large volume change during the lithiation/de-lithiation process (Lao et al., 2017). The volume expansion could cause structural pulverization and rapid capacity decay, limiting their practical applications (Obrovac and Chevrier, 2014). Liu and co-workers recently reported the use of tincone, at the deposited state by MLD, as a stable and high-capacity anode in LIBs (Zhu et al., 2020). Tincone was deposited at $150^{\circ} \mathrm{C}$ by MLD using tetrakis(dimethylamino)tin(IV) (TDMASn) and GL as the precursors and exhibited a self-limiting growth behavior with a high growth per cycle (GPC) of $2.5 \AA$. The tincone on nitrogendoped carbon nanotubes (tincone/NCNTs) delivered a reversible capacity of $490 \mathrm{mAh} \mathrm{g}^{-1}$ for 100 cycles and excellent rate capability of $432.7,368.7,301.0,242.1$, and $173.2 \mathrm{mAh} \mathrm{g}^{-1}$, at $0.1,0.2,0.5,1.0$, and $2.0 \mathrm{~A} \mathrm{~g}^{-1}$, respectively (Figure 1C), which outperformed both pristine N-CNTs and ALD-deposited $\mathrm{SnO}_{2}$ / CNTs (Zhu et al., 2019a; Zhu et al., 2019b). It was found that the $\mathrm{Sn}$ ions in the unique tincone structure provided high Li-ion storage capacity and acted as redox-active sites, while the surrounding organic species served as a flexible matrix to accommodate the volume change of Sn during the cycling. This work not only provides a stable MLD anode for fabricating $3 \mathrm{D}$ microbatteries, but also points out a new route to address the long-standing problems in alloying-type anodes by engineering the material structure at the molecular level (such as Si-based metalcone).

Recently, Karppinen and co-workers made encouraging progress on MLD surface chemistry and process development for Li-containing organic-inorganic thin films ( $\mathrm{Li}$ dicarboxylates), and demonstration of these novel materials as the anode [Li-TPA (Nisula and Karppinen, 2016), Li-PDC, LiNDC, Li-BPDC, and Li-ZAO (Multia et al., 2020)] and the cathode $\left(\mathrm{Li}_{2} \mathrm{Q}\right)$ (Nisula and Karppinen, 2018) in LIBs. For the first time, they reported the MLD recipes for these $\mathrm{Li}^{-}$ dicarboxylates, by using lithium bis(trimethylsilyl)amide (LiHMDS) as the $\mathrm{Li}$ precursor, in combination with five organic linkers, terephthalic acid (TPA), pyridinedicarboxylic acid (PDC), 2,6-naphthalenedicarboxylic acid (NDC), 4,4' biphenydicarboxylic acid (BPDC), and 4, $4^{\prime}$ azobenzenedicarboxylic acid (AZO) (Figure 1D). Due to the different organic precursors employed, Li-TPA, Li-PDC, LiNDC, Li-BPDC, and Li-ZAO exhibited a GPC of 3.0, 2.5, 2.3, 7.0, and 7.0 $\AA$, respectively, (Table 1). Moreover, these MLD processes yielded highly crystalline and air-stable thin films at low deposition temperatures of $220-240^{\circ} \mathrm{C}$. Furthermore, galvanostatic cycling experiments verified the electrochemical activity of these novel films toward Li-ion storage. The redox reaction mechanisms for Li-TPA and LZO are shown in Figure 1D. This series of MLD Li-dicarboxylates is an excellent example of engineering MLD material structures by adjusting the organic precursors and thus tailoring their electrochemical behaviors toward LIB applications. This strategy might be extended to other hybrid organic-inorganic materials by MLD.

In addition to the anode, cathode materials with hybrid organic-inorganic structures have been synthesized by MLD. Dilithium-1,4benzenediolate $\left(\mathrm{Li}_{2} \mathrm{Q}\right)$ was successfully deposited at $160^{\circ} \mathrm{C}$ by MLD using LiHMDS and hydroquinone (HQ) as the precursors (Nisula and Karppinen, 2018). Compared with the known organic electrode materials (p-benzoquinone), MLD achieved in-situ incorporation of $\mathrm{Li}$ into the as-deposited $\mathrm{Li}_{2} \mathrm{Q}$ 
film and therefore, can be directly used as the cathode in full cells due to its lithiated state. The authors further fabricated a solidstate $\mathrm{Li}_{2} \mathrm{Q} / \mathrm{LiPON} / \mathrm{Cu}$ cell using Pt as the current collector, ALDLiPON as the solid electrolyte $(30 \mathrm{~nm})$, and $\mathrm{Li}_{2} \mathrm{Q}$ as the cathode (5-42 nm) (Figure 1E). Cyclic voltammetry (CV) testing revealed a pair of redox peaks at $3.38 \mathrm{~V}$ (oxidation) and $2.57 \mathrm{~V}$ (reduction) at $0.2 \mathrm{mV} \mathrm{s}^{-1}$. Due to thin electrode design, the solid-state cell exhibited ultrahigh rate capabilities and could reach $50 \%$ of the full capacity in less than $0.25 \mathrm{~s}$, with energy and power densities of $108 \mathrm{mWh} \mathrm{cm}^{-3}$ and $508 \mathrm{w} \mathrm{cm}^{-3}$, respectively. Furthermore, a proof-of-concept all-ALD/MLD-made organic battery was fabricated by using $\mathrm{Li}_{2} \mathrm{Q}$ cathode, LiPON solid electrolyte, and Li-TPA anode (Nisula and Karppinen, 2018), demonstrating the feasibility of ALD and/or MLD in the design of 3D microbatteries.

Solid electrolyte thin films have been limited to inorganic materials (Sheil and Chang, 2020) until very recently, Dasgupta et al. reported Li-ion conducting "lithicone" by MLD (Kazyak et al., 2020). The lithicone was deposited at $135^{\circ} \mathrm{C}$ by sequentially introducing lithium tert-butoxide $\left(\mathrm{LiO}^{t} \mathrm{Bu}\right)$ and $\mathrm{EG}$ (Figure 1F). Both precursors were chosen due to their sufficiently high vapor pressure, thermal stability and demonstrated self-limiting surface reactions. Lithicone possessed a stoichiometry of $\mathrm{Li}_{1.5} \mathrm{C}_{2} \mathrm{O}_{1.8}$, with a bonding environment distinct from that of lithium carbonate. Most importantly, electrochemical impedance spectroscopy (EIS) measurements showed that lithicone, upon post-annealing at $350^{\circ} \mathrm{C}$, exhibited an ionic conductivity of $3.6 \times 10^{-8} \mathrm{~S} \mathrm{~cm}^{-1}$ at $30^{\circ} \mathrm{C}$ with an activation energy of $0.6 \mathrm{eV}$ (Figure 1F). Chronoamperometry test revealed an electronic conductivity of the film 5-6 orders-of-magnitude lower than the ionic conductivity, making the lithicone suitable as an interlayer or bulk solid electrolyte in thin-film batteries. Although solid polymer electrolytes have been known for many years, this is the first time that organic-containing electrolyte was made in a layer-by-layer fashion by MLD and showed measurable ionic conductivity.

The above recent works positively proved the possibility and versatility of using the MLD technique to fabricate multifunctional hybrid organic-inorganic anode, cathode, and solid electrolyte with targeted applications in rechargeable batteries. Overall, it can be found that the use of organic precursors during the MLD processes adds extra, and sometimes surprising, functionalities into these novel thin-film materials to address existing challenges and open new opportunities.

\section{OTHER NEW MLD THIN FILMS PROMISING FOR RECHARGEABLE BATTERIES}

In addition to the aforementioned materials, several new MLD thin films have been developed recently with the potential for battery applications. This group of new MLD thin films includes magnesicone (Kint et al., 2020), manganicone (Ahvenniemi and Karppinen, 2016), lithium propane dioxide (Wang et al., 2020), Ti-based maleic acid (Cao et al., 2019), and Mn- and Co-based hybrid films (Ahvenniemi and Karppinen, 2016). The MLD receipts and growth behaviors of these MLD films are summarized in Table 1. It can be found that these new MLD materials show high similarity to other hybrid films that have been validated in LIBs in terms of MLD surface chemistry and film structure, and therefore deserve to be evaluated in electrochemical cells in the future.

There are three main approaches for thoroughly assessing the potential of these new MLD hybrid films in rechargeable batteries. Firstly, these hybrid films, with or without postannealing, might be directly applied as the anode, cathode, or solid electrolyte in LIBs, taking advantage of the electrochemical activity of metal ions. Secondly, the hybrid films could also be employed as surface coating layers to address liquid-solid and solid-solid interface issues in conventional liquid-based LIBs, solid-state LIBs, and next-generation batteries (e.g., $\mathrm{Na}, \mathrm{K}$, and $\mathrm{Zn}$-ion). This surface-engineering approach has been widely demonstrated using alucone, a mature MLD material, in a broad range of applications, such as $\mathrm{Si}$ anode (Piper et al., 2014), Li metal (Zhao et al., 2018a), Na metal (Zhao et al., 2017), Zn metal (He and Liu, 2020), Na cathode (Kaliyappan et al., 2020), and many others (Zhao et al., 2021). Compared to insitu formed SEI, the MLD coating, as an artificial SEI on the electrode, become ion and electron conductive upon contact with charge carriers (e.g., Li-ion, Na-ion), possess better mechanical stability and interfacial structure integrity over repeated cycling, and prevent further side reactions between the electrode and electrolyte. Comprehensive experiment and simulation work has been performed to understand the structure change and role of ALD- $\mathrm{Al}_{2} \mathrm{O}_{3}$ coating in LIBs, but not for MLD-alucone coating yet. Theoretical analysis using density functional theory, ab initio molecular dynamics simulations, and Green's function theory found that the alucone film was composed of Al-O complexes, and $\mathrm{Li}$ atoms bonded to these $\mathrm{O}$ atoms during the lithiation ( $\mathrm{Ma}$ et al., 2015). Once the alucone film was irreversibly saturated with Li atoms, it became electronically conductive. Thirdly, MLD hybrid films (e.g., zincone, magnesicone) have been converted to corresponding highly porous metal oxide materials (e.g., $\mathrm{ZnO}$, $\mathrm{MgO}$ ) by removing the organic backbones via post-annealing (Perrotta et al., 2019a; Perrotta et al., 2019b; Kint et al., 2020). These porous metal oxide structures might be used reactive barrier layers or porous substrates for Li-ion composite solid electrolytes.

\section{SUMMARY AND PERSPECTIVES}

Herein, we discussed the most recent progress in the MLD surface chemistry, process development, and applications of metalcone thin films as active materials in rechargeable LIBs. Several hybrid organic-inorganic anode, cathode, and solid electrolytes have been synthesized by MLD and demonstrated promising electrochemical properties toward Li-ion storage or transport. Moreover, these novel materials have been integrated into allsolid-state thin-film batteries by combining MLD with ALD. Therefore, MLD has shown its great potential in engineering multifunctional thin films for 3D microbattery applications. Nevertheless, challenges remain in MLD and its further applications in energy storage devices and necessitates further 
research into expanding MLD material library, exploiting their use for surface/interface modification in batteries, and developing new precursors and surface chemistries for MLD processes.

\section{Develop New MLD Metalcone Materials}

Despite recent progress in new MLD development, the number of available metalcone materials is still very limited. Further research needs to significantly develop new MLD surface chemistry and process for other metalcone thin films. This could be done by either switching metal-organic precursors that have been validated in ALD and/or adjusting organic reactants to manipulate the film properties. For example, Licontaining organic-inorganic materials might be extended to Naand $\mathrm{K}$-containing ones by changing the $\mathrm{Li}$ precursors $\left(\mathrm{LiO}^{t} \mathrm{Bu}\right.$ and LiHMDS) to their Na- and K-counterparts (Østreng et al., 2014).

\section{Exploit MLD Metalcones in Broad Energy Storage and Conversion Devices}

The possibility of controlling metalcone structure at the molecular level creates tremendous opportunities for various energy storage and conversion systems. Further research might focus on exploring the use of multifunctional metalcones for surface modification purposes in liquid- and solid-state LIBs and other next-generation batteries to improve interfacial stability. Moreover, the metalcones could also be used as supports and over-coating on catalysts to increases their durability and catalytic activities (Gould et al., 2014).

\section{New MLD Precursors and Surface Chemistry}

New metal precursors with high vapor pressure and reactivity are urgently needed to enable the development of more MLD metalcones. For example, although lithicone has been developed, the used $\mathrm{Li}$ precursors $\left(\mathrm{LiO}^{t} \mathrm{Bu}\right)$ are certainly not ideal for the MLD process because they have low vapor pressure and require high sublimation temperatures. This

\section{REFERENCES}

Ahvenniemi, E., and Karppinen, M. (2016). ALD/MLD Processes for Mn and Co Based Hybrid Thin Films. Dalton Trans. 45 (26), 10730-10735. doi:10.1039/ c6dt00851h

Banerjee, A., Wang, X., Fang, C., Wu, E. A., and Meng, Y. S. (2020). Interfaces and Interphases in All-Solid-State Batteries with Inorganic Solid Electrolytes. Chem. Rev. 120 (14), 6878-6933. doi:10.1021/acs.chemrev.0c00101

Bergsman, D. S., Baker, J. G., Closser, R. G., Macisaac, C., Lillethorup, M., Strickler, A. L., et al. (2019). Structurally Stable Manganese Alkoxide Films Grown by Hybrid Molecular Layer Deposition for Electrochemical Applications. Adv. Funct. Mater. 29 (43), 1904129. doi:10.1002/adfm.201904129

Cao, Y.-Q., Zhang, W., Xu, L., Liu, C., Zhu, L., Wang, L.-G., et al. (2019). Growth Mechanism, Ambient Stability, and Charge Trapping Ability of Ti-Based Maleic Acid Hybrid Films by Molecular Layer Deposition. Langmuir 35 (8), 3020-3030. doi:10.1021/acs.langmuir.8b04137

Chen, L., Huang, Z., Shahbazian-Yassar, R., Libera, J. A., Klavetter, K. C., Zavadil, K. R., et al. (2018). Directly Formed Alucone on Lithium Metal challenge becomes even severe in $\mathrm{Na}$ and $\mathrm{K}$ precursors (Østreng et al., 2014). A further advance in MLD would be benefited from the design of new precursor chemistry to satisfy self-limiting and complete growth reactions.

\section{Overcome Shortcomings of MLD for Practical Applications}

Many MLD thin films tend to be unstable in contact with water and oxygen and might experience immediate changes in the surface structure upon exposure to the ambient environment (Liang et al., 2009; Van De Kerckhove et al., 2018). It remains unknown how this surface change affects the properties of MLD coating on the electrode. The scalability of MLD is yet to be demonstrated using fluidized-bed or roll-to-roll equipment. In particular, the organic precursor is more "sticky" than water during the low-temperature deposition process and likely to require more purging time to remove physically absorbed precursors. There is little knowledge in scalable MLD and potential issues, which deserve further investigation.

\section{AUTHOR CONTRIBUTIONS}

JL and JW conceived the idea. JL wrote the manuscript, and JW revised it. All authors discussed the topics and contributed to the organization of this paper.

\section{FUNDING}

This work was supported by the Nature Sciences and Engineering Research Council of Canada (NSERC), Canada Foundation for Innovation (CFI), BC Knowledge Development Fund (BCKDF), and the University of British Columbia (UBC). JW's work was supported by the start-up fund and "Young Scientist Studio" of Harbin Institute of Technology, National Natural Science Foundation of China (No. U1932205, No. 22075063), Natural Science Funds of Heilongjiang Province (No. ZD2019B001).

for High-Performance Li Batteries and Li-S Batteries with High Sulfur Mass Loading. ACS Appl. Mater. Inter. 10 (8), 7043-7051. doi:10.1021/acsami. $7 \mathrm{~b} 15879$

Chen, Z., Qin, Y., Amine, K., and Sun, Y.-K. (2010). Role of Surface Coating on Cathode Materials for Lithium-Ion Batteries. J. Mater. Chem. 20 (36), 7606. doi:10.1039/c0jm00154f

George, S. M., Yoon, B., and Dameron, A. A. (2009). Surface Chemistry for Molecular Layer Deposition of Organic and Hybrid Organic-Inorganic Polymers. Acc. Chem. Res. 42 (4), 498-508. doi:10.1021/ar800105q

Gould, T. D., Izar, A., Weimer, A. W., Falconer, J. L., and Medlin, J. W. (2014). Stabilizing Ni Catalysts by Molecular Layer Deposition for Harsh, Dry Reforming Conditions. ACS Catal. 4 (8), 2714-2717. doi:10.1021/cs500809w

He, H., and Liu, J. (2020). Suppressing Zn Dendrite Growth by Molecular Layer Deposition to Enable Long-Life and Deeply Rechargeable Aqueous Zn Anodes. J. Mater. Chem. A. 8 (42), 22100-22110. doi:10.1039/d0ta07232j

Heiska, J., Nisula, M., Rautama, E.-L., Karttunen, A. J., and Karppinen, M. (2020). Atomic/molecular Layer Deposition and Electrochemical Performance of Dilithium 2-aminoterephthalate. Dalton Trans. 49 (5), 1591-1599. doi:10. 1039/c9dt04572d 
Kaliyappan, K., Or, T., Deng, Y. P., Hu, Y., Bai, Z., and Chen, Z. (2020). Constructing Safe and Durable High-Voltage P2 Layered Cathodes for Sodium Ion Batteries Enabled by Molecular Layer Deposition of Alucone. Adv. Funct. Mater. 30 (17), 1910251. doi:10.1002/adfm.201910251

Kazyak, E., Chen, K.-H., Wood, K. N., Davis, A. L., Thompson, T., Bielinski, A. R., et al. (2017). Atomic Layer Deposition of the Solid Electrolyte Garnet Li7La3Zr2O12. Chem. Mater. 29 (8), 3785-3792. doi:10.1021/acs.chemmater. $7 \mathrm{~b} 00944$

Kazyak, E., Shin, M., Lepage, W. S., Cho, T. H., and Dasgupta, N. P. (2020). Molecular Layer Deposition of Li-Ion Conducting "Lithicone" Solid Electrolytes. Chem. Commun. 56 (99), 15537-15540. doi:10.1039/d0cc06077a

Kint, J., Mattelaer, F., Vandenbroucke, S. S. T., Muriqi, A., Minjauw, M. M., Nisula, M., et al. (2020). Molecular Layer Deposition of "Magnesicone", a MagnesiumBased Hybrid Material. Chem. Mater. 32 (11), 4451-4466. doi:10.1021/acs. chemmater.9b05116

Knoops, H. C. M., Donders, M. E., Van De Sanden, M. C. M., Notten, P. H. L., and Kessels, W. M. M. (2012). Atomic Layer Deposition for Nanostructured Li-Ion Batteries. J. Vacuum Sci. Tech. A: Vacuum, Surf. Films 30 (1), 010801. doi:10. 1116/1.3660699

Lao, M., Zhang, Y., Luo, W., Yan, Q., Sun, W., and Dou, S. X. (2017). Alloy-based Anode Materials toward Advanced Sodium-Ion Batteries. Adv. Mater. 29 (48), 1700622. doi:10.1002/adma.201700622

Lee, B. H., Yoon, B., Abdulagatov, A. I., Hall, R. A., and George, S. M. (2013). Growth and Properties of Hybrid Organic-Inorganic Metalcone Films Using Molecular Layer Deposition Techniques. Adv. Funct. Mater. 23 (5), 532-546. doi:10.1002/adfm.201200370

Liang, X., Yu, M., Li, J., Jiang, Y.-B., and Weimer, A. W. (2009). Ultra-thin Microporous-Mesoporous Metal Oxide Films Prepared by Molecular Layer Deposition (MLD). Chem. Commun. 14 (46), 7140. doi:10.1039/b911888h

Liu, H., Zhang, G., Zheng, X., Chen, F., and Duan, H. (2020). Emerging Miniaturized Energy Storage Devices for Microsystem Applications: from Design to Integration. Int. J. Extrem. Manuf. 2 (4), 042001. doi:10.1088/ 2631-7990/abba12

Liu, J., Zhu, H., and Shiraz, M. H. A. (2018). Toward 3D Solid-State Batteries via Atomic Layer Deposition Approach. Front. Energ. Res. 6. doi:10.3389/fenrg. 2018.00010

Lotfabad, E. M., Kalisvaart, P., Kohandehghan, A., Cui, K., Kupsta, M., Farbod, B., et al. (2014). Si Nanotubes ALD Coated with TiO2, TiN or Al2O3as High Performance Lithium Ion Battery Anodes. J. Mater. Chem. A. 2 (8), 2504-2516. doi:10.1039/c3ta14302c

Ma, Y., Martinez De La Hoz, J. M., Angarita, I., Berrio-Sanchez, J. M., Benitez, L., Seminario, J. M., et al. (2015). Structure and Reactivity of Alucone-Coated Films on Si and Lix SiySurfaces. ACS Appl. Mater. Inter. 7 (22), 11948-11955. doi:10. 1021/acsami.5b01917

Meng, X. (2017). An Overview of Molecular Layer Deposition for Organic and Organic-Inorganic Hybrid Materials: Mechanisms, Growth Characteristics, and Promising Applications. J. Mater. Chem. A. 5 (35), 18326-18378. doi:10.1039/c7ta04449f

Multia, J., Heiska, J., Khayyami, A., and Karppinen, M. (2020). Electrochemically Active In Situ Crystalline Lithium-Organic Thin Films by ALD/MLD. ACS Appl. Mater. Inter. 12 (37), 41557-41566. doi:10.1021/acsami.0c11822

Nisula, M., and Karppinen, M. (2016). Atomic/Molecular Layer Deposition of Lithium Terephthalate Thin Films as High Rate Capability Li-Ion Battery Anodes. Nano Lett. 16 (2), 1276-1281. doi:10.1021/acs.nanolett.5b04604

Nisula, M., and Karppinen, M. (2018). In Situ lithiated Quinone Cathode for ALD/ MLD-fabricated High-Power Thin-Film Battery. J. Mater. Chem. A. 6 (16), 7027-7033. doi:10.1039/c8ta00804c

Obrovac, M. N., and Chevrier, V. L. (2014). Alloy Negative Electrodes for Li-Ion Batteries. Chem. Rev. 114 (23), 11444-11502. doi:10.1021/cr500207g

Østreng, E., Sønsteby, H. H., Øien, S., Nilsen, O., and Fjellvåg, H. (2014). Atomic Layer Deposition of Sodium and Potassium Oxides: Evaluation of Precursors and Deposition of Thin Films. Dalton Trans. 43 (44), 16666-16672. doi:10. 1039/c4dt01930j

Oudenhoven, J. F. M., Baggetto, L., and Notten, P. H. L. (2011). All-Solid-State Lithium-Ion Microbatteries: A Review of Various Three-Dimensional Concepts. Adv. Energ. Mater. 1 (1), 10-33. doi:10.1002/aenm.201000002

Pearse, A. J., Schmitt, T. E., Fuller, E. J., El-Gabaly, F., Lin, C.-F., Gerasopoulos, K., et al. (2017). Nanoscale Solid State Batteries Enabled by Thermal Atomic Layer
Deposition of a Lithium Polyphosphazene Solid State Electrolyte. Chem. Mater. 29 (8), 3740-3753. doi:10.1021/acs.chemmater.7b00805

Pearse, A., Schmitt, T., Sahadeo, E., Stewart, D. M., Kozen, A., Gerasopoulos, K., et al. (2018). Three-Dimensional Solid-State Lithium-Ion Batteries Fabricated by Conformal Vapor-phase Chemistry. ACS Nano 12 (5), 4286-4294. doi:10. 1021/acsnano.7b08751

Perrotta, A., Berger, R., Muralter, F., and Coclite, A. M. (2019a). Mesoporous ZnO Thin Films Obtained from Molecular Layer Deposited “Zincones”. Dalton Trans. 48 (37), 14178-14188. doi:10.1039/c9dt02824b

Perrotta, A., Pilz, J., Pachmajer, S., Milella, A., and Coclite, A. M. (2019b). On the Transformation of "Zincone"-like into Porous $\mathrm{ZnO}$ Thin Films from Subsaturated Plasma Enhanced Atomic Layer Deposition. Beilstein J. Nanotechnol. 10, 746-759. doi:10.3762/bjnano.10.74

Piper, D. M., Travis, J. J., Young, M., Son, S.-B., Kim, S. C., Oh, K. H., et al. (2014). Reversible High-Capacity Si Nanocomposite Anodes for Lithium-Ion Batteries Enabled by Molecular Layer Deposition. Adv. Mater. 26 (10), 1596-1601. doi:10.1002/adma.201304714

Roberts, M., Johns, P., Owen, J., Brandell, D., Edstrom, K., El Enany, G., et al. (2011). 3D Lithium Ion Batteries-From Fundamentals to Fabrication. J. Mater. Chem. 21 (27), 9876. doi:10.1039/c0jm04396f

Rowden, B., and Garcia-Araez, N. (2020). A Review of Gas Evolution in Lithium Ion Batteries. Energ. Rep. 6, 10-18. doi:10.1016/j.egyr.2020.02.022

Scott, I. D., Jung, Y. S., Cavanagh, A. S., Yan, Y., Dillon, A. C., George, S. M., et al. (2011). Ultrathin Coatings on Nano-LiCoO2for Li-Ion Vehicular Applications. Nano Lett. 11 (2), 414-418. doi:10.1021/nl1030198

Sheil, R., and Chang, J. P. (2020). Synthesis and Integration of Thin Film Solid State Electrolytes for 3D Li-Ion Microbatteries. J. Vacuum Sci. Tech. A 38 (3), 032411. doi:10.1116/1.5142859

Son, S.-B., Wang, Y., Xu, J., Li, X., Groner, M., Stokes, A., et al. (2017). Systematic Investigation of the Alucone-Coating Enhancement on Silicon Anodes. ACS Appl. Mater. Inter. 9 (46), 40143-40150. doi:10.1021/acsami.7b08960

Sundberg, P., and Karppinen, M. (2014). Organic and Inorganic-Organic Thin Film Structures by Molecular Layer Deposition: A Review. Beilstein J. Nanotechnol. 5, 1104-1136. doi:10.3762/bjnano.5.123

Van De Kerckhove, K., Barr, M. K. S., Santinacci, L., Vereecken, P. M., Dendooven, J., and Detavernier, C. (2018). The Transformation Behaviour of "Alucones", Deposited by Molecular Layer Deposition, in Nanoporous Al2O3 Layers. Dalton Trans. 47 (16), 5860-5870. doi:10.1039/c8dt00723c

Van De Kerckhove, K., Mattelaer, F., Deduytsche, D., Vereecken, P. M., Dendooven, J., and Detavernier, C. (2016). Molecular Layer Deposition of "titanicone", a Titanium-Based Hybrid Material, as an Electrode for Lithium-Ion Batteries. Dalton Trans. 45 (3), 1176-1184. doi:10.1039/ c5dt03840e

Van De Kerckhove, K., Mattelaer, F., Dendooven, J., and Detavernier, C. (2017). Molecular Layer Deposition of "vanadicone", a Vanadium-Based Hybrid Material, as an Electrode for Lithium-Ion Batteries. Dalton Trans. 46 (14), 4542-4553. doi: 10.1039/c7dt00374a

Wang, H., Gregorczyk, K. E., Lee, S. B., Rubloff, G. W., and Lin, C.-F. (2020). LiContaining Organic Thin Film-Structure of Lithium Propane Dioxide via Molecular Layer Deposition. J. Phys. Chem. C 124 (12), 6830-6837. doi:10. 1021/acs.jpcc.9b11868

Weimer, A. W. (2019). Particle Atomic Layer Deposition. J. Nanopart Res. 21 (1). doi:10.1007/s11051-018-4442-9

Xiao, X., Lu, P., and Ahn, D. (2011). Ultrathin Multifunctional Oxide Coatings for Lithium Ion Batteries. Adv. Mater. 23 (34), 3911-3915. doi:10.1002/adma. 201101915

Xu, L., Tang, S., Cheng, Y., Wang, K., Liang, J., Liu, C., et al. (2018). Interfaces in SolidState Lithium Batteries. Joule 2 (10), 1991-2015. doi:10.1016/j.joule.2018.07.009

Yan, P., Zheng, J., Liu, J., Wang, B., Cheng, X., Zhang, Y., et al. (2018). Tailoring Grain Boundary Structures and Chemistry of Ni-Rich Layered Cathodes for Enhanced Cycle Stability of Lithium-Ion Batteries. Nat. Energ. 3 (7), 600-605. doi:10.1038/s41560-018-0191-3

Zhao, Y., Goncharova, L. V., Sun, Q., Li, X., Lushington, A., Wang, B., et al. (2018a). Robust Metallic Lithium Anode Protection by the Molecular-LayerDeposition Technique. Small Methods 2 (5), 1700417. doi:10.1002/smtd. 201700417

Zhao, Y., Goncharova, L. V., Zhang, Q., Kaghazchi, P., Sun, Q., Lushington, A., et al. (2017). Inorganic-Organic Coating via Molecular Layer Deposition 
Enables Long Life Sodium Metal Anode. Nano Lett. 17 (9), 5653-5659. doi:10. 1021/acs.nanolett.7b02464

Zhao, Y., Zhang, L., Liu, J., Adair, K., Zhao, F., Sun, Y., et al. (2021). Atomic/ molecular Layer Deposition for Energy Storage and Conversion. Chem. Soc. Rev. 50, 3889-3956. doi:10.1039/d0cs00156b

Zhao, Y., Zheng, K., and Sun, X. (2018b). Addressing Interfacial Issues in LiquidBased and Solid-State Batteries by Atomic and Molecular Layer Deposition. Joule 2 (12), 2583-2604. doi:10.1016/j.joule.2018.11.012

Zhu, H., Aboonasr Shiraz, M. H., Yao, L., Adair, K., Wang, Z., Tong, H., et al. (2020). Molecular-layer-deposited Tincone: a New Hybrid Organic-Inorganic Anode Material for Three-Dimensional Microbatteries. Chem. Commun. 56 (86), 13221-13224. doi:10.1039/d0cc03869e

Zhu, S., Liu, J., and Sun, J. (2019a). Growth of Ultrathin SnO2 on Carbon Nanotubes by Atomic Layer Deposition and Their Application in Lithium Ion Battery Anodes. Appl. Surf. Sci. 484, 600-609. doi:10.1016/j.apsusc.2019.04.163
Zhu, S., Liu, J., and Sun, J. (2019b). Precise Growth of Al2O3/SnO2/CNTs Composites by a Two-step Atomic Layer Deposition and Their Application as an Improved Anode for Lithium Ion Batteries. Electrochimica Acta 319, 490-498. doi:10.1016/j.electacta.2019.07.027

Conflict of Interest: The authors declare that the research was conducted in the absence of any commercial or financial relationships that could be construed as a potential conflict of interest.

Copyright $\odot 2021$ Liu and Wang. This is an open-access article distributed under the terms of the Creative Commons Attribution License (CC BY). The use, distribution or reproduction in other forums is permitted, provided the original author(s) and the copyright owner(s) are credited and that the original publication in this journal is cited, in accordance with accepted academic practice. No use, distribution or reproduction is permitted which does not comply with these terms. 